\title{
Conhecimentos sobre sexualidade: Construção e validação de um instrumento de avaliação para adolescentes em contexto escolar
}

\author{
Cristiana Pereira de Carvalhoi, Maria do Rosário Moura \\ Pinheiroii \& José Pinto Gouveiaiii \\ Universidade de Coimbra, Portugal \\ Duarte Rei Vilariv \\ Universidade Lusíada de Lisboa, Portugal
}

Resumo

O conhecimento, enquanto fator capaz de exercer influência sobre as atitudes, é um pré-requisito fundamental nos programas de prevenção dos comportamentos sexuais de risco. Este estudo teve como objetivo desenvolver um instrumento para avaliar os conhecimentos dos adolescentes sobre a sexualidade. O Questionário de Conhecimentos sobre Sexualidade pretende avaliar os conhecimentos num continuum de dificuldade e distinguir os alunos com diferentes níveis de saberes. A amostra foi constituída por 1545 adolescentes com uma média de 16 anos $(D P=1.80)$ que frequentam a escola. Os resultados evidenciam que as raparigas estão mais informadas do que os rapazes, e que os jovens portugueses com mais de 15 anos, sem religião, com mais de 5 anos de educação sexual na escola e que tiveram a mãe como educadora sexual são os que têm mais conhecimentos sobre a sexualidade. As atitudes dos adolescentes em relação ao preservativo mostraram estar associadas aos conhecimentos e os adolescentes que usaram preservativo no último encontro sexual revelaram níveis mais elevados de conhecimentos.

Palavras-chave

Conhecimentos; Educação Sexual; Adolescentes; Questionário 


\section{Introdução ${ }^{1}$}

O acesso à educação e à informação sobre a sexualidade, enquanto direito sexual (International Planned Parenthood Federation [IPPF], 2007, 2008; World Health Organization [WHO], 2010), é considerado indispensável na prevenção dos comportamentos sexuais de risco, em particular junto da população jovem (Lei n. 60/2009; Ministério da Educação, 2000; Portaria n. 196-A/2010; WHO, 2010). Segundo a Organização Mundial de Saúde (OMS), a realização de uma educação sexual holística fornece a crianças e jovens informação cientificamente correta sobre todos os aspetos da sexualidade e, ao mesmo tempo, ajuda-os a desenvolver as habilidades para agir em conformidade com essa informação (WHO, 2010). De acordo com a literatura, a ausência de conhecimentos nesta área pode ter implicações negativas na tomada de decisão e na realização de escolhas pouco informadas, corretas e seguras (Farih, Khan, Freeth, \& Meads, 2014; IPPF, 2007) em termos de saúde sexual e reprodutiva.

Embora o conhecimento por si só não garanta a modificação dos comportamentos (Fisher \& Fisher, 1992; Kalichman et al., 2002), este é considerado um pré-requisito fundamental nos programas de prevenção dos comportamentos sexuais de risco (Degroote, Vogelaers, Liefhooghe, Vermeir, \& Vandijck, 2014). Estudos apontam para que os conhecimentos possam exercer influência sobre as atitudes dos sujeitos, podendo contribuir para a diminuição de comportamentos de risco (Reis \& Matos, 2007; Roque, 2001; Synovitz, Herbert, Kelley, \& Carlson, 2002). Jovens com elevados conhecimentos sobre a sexualidade tendem a não aceitar correr riscos, enquanto os que possuem menos conhecimentos aceitam mais facilmente e expõem-se mais a situações de risco (Reis \& Matos, 2007). Além disso, jovens com elevado conhecimento nos temas da sexualidade reportam uma vivência mais gratificante da sexualidade, demonstram uma maior capacidade de pedir ajuda (quando necessário) e tendem a apresentar mais comportamentos preventivos, especificamente no que diz respeito ao uso consistente do preservativo (Vilar \& Ferreira, 2009). Neste sentido, uma das condições para alcançar a redução do número de casos de jovens infetados com o HIVISIDA consiste em ter um bom conhecimento (informação) sobre a transmissão de infeções sexualmente transmissíveis (IST), em particular do HIV/SIDA (Degroote et al., 2014). 
Por ser considerado um fator importante na alteração comportamental, o conhecimento sobre o $\mathrm{VIH} / \mathrm{SIDA}$ representa uma das variáveis mais estudadas na literatura (Ramiro, 2013). Se os jovens tiverem conhecimentos, motivação, atitudes positivas e aptidões comportamentais para a adotação de comportamentos sexuais seguros (Martins, 2010; Reis, Ramiro, Matos, \& Diniz, 2013) poderão mais facilmente alterar comportamentos.

Embora os adolescentes tenham facilidade em aceder à informação sobre sexualidade por meio de fontes diversas (como os amigos, os media e a Internet), nem sempre esta informação é a mais correta, atual e completa (Matos, 2008; Ramiro, Reis, Matos, Diniz, \& Simões, 2011). Segundo estudos portugueses, realizados no âmbito dos comportamentos sexuais, existem lacunas no conhecimento dos adolescentes e jovens estudantes em relação a aspetos preventivos (Matos, Ramiro, Reis, \& Equipa Aventura Social, 2013; Vilar \& Ferreira, 2009). A pesquisa realizada por Vilar e Ferreira (2009), com 2621 adolescentes portugueses, revelou reduzidos conhecimentos sobre potenciais situações de risco de gravidez não desejada, falta de informação em alguns aspetos preventivos sobre contraceção e IST (à exceção do HIV/SIDA) e, ainda, ausência de conhecimentos sobre prevenção de riscos associados à sexualidade e à saúde. Níveis inferiores de conhecimentos sobressaem entre os rapazes (Vilar \& Ferreira, 2009), tal como referem outros estudos nacionais (Matos et al., 2013). Os autores encontraram uma associação positiva entre níveis elevados de conhecimentos e o uso consistente do preservativo e outros métodos contracetivos (Vilar \& Ferreira, 2009), o que reforça a sua importância nos programas de prevenção específicos para reduzir a transmissão do HIV/SIDA em adolescentes e jovens adultos.

Segundo o estudo sobre a sexualidade dos jovens portugueses entre os 13 e os 21 anos (Online Study of Young People's Sexuality [OSYS], 2011) desenvolvido pela Equipa da Aventura Social, existe um nível de conhecimentos insatisfatório nesta população em relação à pílula contracetiva, à pilula do dia seguinte, mas razoável face às IST e aos modos de transmissão do VIH/SIDA (Matos et al., 2013). Apenas 69.8\% dos jovens consideraram o preservativo 0 melhor método de prevenção contra as infeções sexualmente transmissíveis, com as raparigas a evidenciarem níveis mais elevados de conhecimentos em todas as áreas (Matos et al., 2013). 
Ambos gostariam de saber mais sobre contraceção, sexo seguro, IST e segurança pessoal (Matos et al., 2013).

Apesar de os adolescentes considerarem satisfatório o conhecimento que possuem sobre a sexualidade (Matos et al., 2013; Matos, Reis, Ramiro, \& Equipa Aventura Social, 2011), os estudos apontam necessidades de (in)formação e esclarecimento (reportadas pelos próprios jovens) em assuntos relacionados com os comportamentos sexuais de risco e proteção (Carvalho \& Pinheiro, 2013), como IST, contraceção, sexo seguro, segurança pessoal, prazer e orgasmo (Matos et al., 2011), e evidenciam problemas no domínio do conhecimento, principalmente entre os adolescentes que não tiveram educação sexual na escola (Ramiro, Reis, Matos, \& Diniz, 2011; Vilar \& Ferreira, 2009).

Considerando que a educação sexual em meio escolar tem evidenciado um impacto positivo ao nível dos conhecimentos, atitudes e comportamentos sexuais de proteção dos estudantes (Kirby, Laris, \& Rolleri, 2007; Matos, 2008; Paulos \& Valadas, 2015; Ramiro, 2013; Vilar \& Ferreira, 2009), torna-se cada vez mais necessário proceder a uma avaliação de diagnóstico dos conhecimentos que os adolescentes possuem, para o planeamento de intervenções capazes de promover a adoção e manutenção de comportamentos preventivos (UNESCO, 2010). Aumentar os conhecimentos e substituir as crenças dos adolescentes por informação cientificamente correta são aspetos que merecem atenção da comunidade escolar envolvida nos programas de promoção da saúde sexual e reprodutiva (Sánchez, 1990; UNESCO, 2010; WHO, 2010). Neste sentido, a produção de instrumentos consistentes, capazes de apoiar a avaliação dos programas de educação sexual, em termos de identificação de conhecimentos (o que os adolescentes sabem, não sabem e precisam saber) e de diagnóstico de necessidades de formação dos/as alunos/as nesta área, revela-se de grande utilidade para as escolas portuguesas.

Assim, este estudo teve como objetivo desenvolver um instrumento que permita realizar a avaliação dos conhecimentos dos adolescentes sobre a sexualidade. Para isso, foi construído o Questionário de Conhecimentos sobre Sexualidade (QCS). Os conhecimentos dos adolescentes foram estudados considerando variáveis pessoais (género, idade, escolaridade, religião, nacionalidade e área de residência) e educacionais (escolares e 
familiares). Foi também analisada a relação dos conhecimentos dos adolescentes portugueses sobre a sexualidade com as suas atitudes e comportamentos de prevenção face ao VIH/SIDA.

\section{Método}

\section{Participantes}

A amostra do estudo é constituída por 1545 adolescentes, dos quais $51.1 \%$ ( $n=790)$ são rapazes e $48.9 \%$ ( $n=755)$ são raparigas, com uma média de idades de 16 anos $(\mathrm{M}=16.44, \mathrm{DP}=1.80)$. A maioria dos adolescentes é de nacionalidade portuguesa $(94.7 \%, n=1447)$ e considera-se religiosa $(63 \%$, $n=965)$. No que diz respeito ao ano de escolaridade, $23.6 \% \quad(n=365)$ frequentam o $3^{\circ}$ Ciclo $\left(7^{\circ}, 8^{\circ}\right.$ e $9^{\circ}$ anos) e $76.3 \%$ ( $n=1180$ ) frequentam o ensino secundário $\left(10^{\circ}, 11^{\circ}\right.$ e $12^{\circ}$ anos), e residem nas regiões Centro e Lisboa e Vale do Tejo, em meio rural (68.3\%; $n=1042)$ e urbano $(31.7 \% ; n=483)$.

\section{Instrumento}

\section{Processo de construção e aperfeiçoamento do questionário}

$O$ instrumento de avaliação utilizado no estudo, denominado por Questionário de Conhecimentos sobre Sexualidade (QCS), foi desenvolvido pela equipa de investigadores desta pesquisa (Anexo). Para avaliar o grau de conhecimentos dos adolescentes acerca da sexualidade, foram elaborados 26 itens que pretendem ilustrar os conhecimentos em seis áreas temáticas da educação sexual. A elaboração destes itens, integrados nestas áreas da sexualidade, baseou-se no quadro teórico de referência presente na Matriz de conteúdos de Educação Sexual proposta pela Organização Mundial de Saúde (WHO, 2010) e nos conteúdos mínimos de educação sexual propostos pela Legislação Portuguesa (Portaria n. ${ }^{\circ}$ 196-A/2010) (Quadro 1).

Embora os temas 2 e 6 não estejam contemplados nos conteúdos mínimos de abordagem da sexualidade em meio escolar (Portaria n. ${ }^{\circ} 196-$ A/2010) (Quadro 1), consideramos as recomendações da OMS, correspondentes às diretrizes para o desenvolvimento de uma educação sexual integral e holística a nível Europeu (WHO, 2010). Além disso, foi do nosso interesse ultrapassar a abordagem tradicional da educação sexual centrada nos potenciais riscos da sexualidade, como a gravidez indesejada e 
as IST (WHO, 2010), mas também considerá-la numa perspetiva positiva e promotora de prazer e bem-estar. Importa também referir que, na legislação portuguesa, a abordagem do tema 4 refere-se ao "conhecimento das taxas e tendências de maternidade e da paternidade na adolescência ${ }^{2}$ e compreensão do respetivo significado" (Portaria n. $\left.{ }^{\circ} 196-A / 2010\right)$, sendo que o conteúdo dos itens no QCS afastou-se deste objetivo. Os itens do questionário que compõem esta área temática são de natureza preventiva.

No processo de construção e aperfeiçoamento da escala, optámos por submeter o questionário, na primeira etapa, à apreciação de dois júris com conhecimento na área, que avaliaram a pertinência das dimensões definidas, a adequação dos itens às dimensões e a própria formulação dos itens. Foi ainda solicitada aos júris a sua opinião sobre a formulação e adequação de cada um dos itens à população a que se destinava o questionário. As sugestões deram lugar a alterações que se refletiram na primeira versão do questionário. Posteriormente, o questionário foi analisado por 35 pedagogos/as (33 do género feminino) a frequentar a disciplina de Educação para a Saúde do Mestrado em Ciências da Educação ( $1^{\circ}$ ano do $2^{\circ}$ Ciclo de Estudos) da Faculdade de Psicologia e de Ciências da Educação da Universidade de Coimbra. O objetivo foi recolher a sua opinião em relação a alguns aspetos do questionário, nomeadamente: grau de facilidade/ dificuldade, adequação das instruções, tempo médio de aplicação do questionário, qualidade dos itens relativamente à linguagem (simplicidade, clareza, compreensão e ambiguidade), ao conteúdo (desejabilidade social) e à apresentação e formato de resposta aos itens (verdadeiro ou falso), considerando o seu conhecimento relativamente ao grupo alvo a que se destina o instrumento. Foram introduzidas algumas alterações pontuais e foi elaborada a versão final do questionário (Quadro 1). 
Quadro 1 - Correspondência da matriz de conteúdos de educação sexual da OMS (WHO, 2010) e dos conteúdos mínimos de educação sexual propostos na Portaria $\mathrm{n} .{ }^{\circ} 196-\mathrm{A} / 2010 \mathrm{com}$ as seis áreas temáticas do QCS

\begin{tabular}{|c|c|c|c|c|c|}
\hline \multirow{2}{*}{$\begin{array}{c}\text { Áreas temáticas } \\
\text { do QCS }\end{array}$} & \multicolumn{3}{|c|}{ Matriz da OMS } & \multicolumn{2}{|c|}{ Portaria n. ${ }^{\circ}$ 196-A/2010 } \\
\hline & Idade & $\begin{array}{l}\text { Categoria } \\
\text { Temática }\end{array}$ & Parâmetro de informação & $\begin{array}{c}\text { Ciclo de } \\
\text { ensino }\end{array}$ & $\begin{array}{c}\text { Conteúdos } \\
\text { Mínimos }\end{array}$ \\
\hline $\begin{array}{l}\text { (1) Primeira } \\
\text { relação sexual e } \\
\text { preocupações } \\
\text { sexuais }\end{array}$ & $12-15$ & Sexualidade & $\cdot 1^{\mathrm{a}}$ experiência sexual. & Secundário & $\begin{array}{l}\text { - Idade de } \\
\text { início das } \\
\text { relações } \\
\text { sexuais. }\end{array}$ \\
\hline \multirow[t]{3}{*}{$\begin{array}{l}\text { (2) Sexualidade e } \\
\text { prazer sexual }\end{array}$} & $12-15$ & Sexualidade & $\begin{array}{l}\text { - Como desfrutar a } \\
\text { sexualidade de forma } \\
\text { adequada (no seu tempo). } \\
\text { - Prazer, masturbação, } \\
\text { orgasmo. }\end{array}$ & \multirow[t]{3}{*}{$\mathrm{X}$} & \multirow[t]{3}{*}{$\mathrm{X}$} \\
\hline & $12-15$ & $\begin{array}{l}\text { Sexualidade, } \\
\text { saúde e bem- } \\
\text { estar }\end{array}$ & $\begin{array}{l}\text { - Influência positiva da } \\
\text { sexualidade na saúde e } \\
\text { bem-estar. }\end{array}$ & & \\
\hline & $\begin{array}{c}+ \text { de } \\
15\end{array}$ & Sexualidade & $\begin{array}{l}\text { - Sexo como mais do que o } \\
\text { coito. }\end{array}$ & & \\
\hline \multirow[t]{2}{*}{$\begin{array}{l}\text { (3) Contraceção } \\
\text { e práticas } \\
\text { sexuais seguras }\end{array}$} & $12-15$ & $\begin{array}{l}\text { Fertilidade e } \\
\text { reprodução }\end{array}$ & $\begin{array}{l}\text { - Factos e mitos } \\
\text { (confiabilidade, } \\
\text { vantagens e desvantagens) } \\
\text { relacionados com os vários } \\
\text { métodos contracetivos } \\
\text { (incluindo a contraceção de } \\
\text { emergência). }\end{array}$ & \multirow[t]{2}{*}{$\begin{array}{l}3^{\circ} \text { Ciclo } \\
\text { Secundário }\end{array}$} & \multirow{2}{*}{$\begin{array}{l}\text { - Compreensão } \\
\text { do uso e } \\
\text { acessibilidade } \\
\text { dos métodos } \\
\text { contracetivos. } \\
\text { - Métodos } \\
\text { contracetivos } \\
\text { disponíveis e } \\
\text { utilizados. }\end{array}$} \\
\hline & & $\begin{array}{l}\text { Sexualidade, } \\
\text { saúde e bem- } \\
\text { estar }\end{array}$ & $\begin{array}{l}\text { - Comportamento de risco } \\
\text { (sexual) e suas } \\
\text { consequências (álcool). }\end{array}$ & & \\
\hline $\begin{array}{l}\text { (4) Prevenção da } \\
\text { Gravidez }\end{array}$ & $12-15$ & $\begin{array}{l}\text { Fertilidade e } \\
\text { reprodução }\end{array}$ & $\begin{array}{l}\text { - Tomada de decisão e } \\
\text { cuidados no caso de } \\
\text { gravidez indesejada. }\end{array}$ & $\begin{array}{l}3^{\circ} \text { Ciclo } \\
\text { Secundário }\end{array}$ & $\begin{array}{l}\text { - Taxas } \\
\text { gravidez. }\end{array}$ \\
\hline \multirow[t]{2}{*}{$\begin{array}{l}\text { (5) Infeções } \\
\text { sexualmente } \\
\text { transmissíveis e } \\
\text { VIH/SIDA }\end{array}$} & $12-15$ & $\begin{array}{l}\text { Sexualidade, } \\
\text { saúde e bem- } \\
\text { estar }\end{array}$ & $\begin{array}{l}\text { - Sintomas, transmissão e } \\
\text { prevenção de IST, incluindo } \\
\text { VIH. }\end{array}$ & $3^{\circ}$ Ciclo & $\begin{array}{l}\text { - Compreensão } \\
\text { da } \\
\text { epidemiologia } \\
\text { das principais } \\
\text { IST (incluindo } \\
\text { infeção por } \\
\text { VIH) e dos } \\
\text { métodos de } \\
\text { prevenção. }\end{array}$ \\
\hline & $\begin{array}{c}+ \text { de } \\
15\end{array}$ & $\begin{array}{l}\text { Sexualidade, } \\
\text { saúde e bem- } \\
\text { estar }\end{array}$ & $\begin{array}{l}\text { - VIH / SIDA e transmissão } \\
\text { de IST, prevenção, } \\
\text { tratamento, cuidados e } \\
\text { apoio. }\end{array}$ & Secundário & $\begin{array}{l}\text { - Doenças e } \\
\text { IST (como } \\
\text { infeção por } \\
\text { VIH) e suas } \\
\text { consequências. } \\
\text { - Prevenção de } \\
\text { IST. }\end{array}$ \\
\hline \multirow[t]{2}{*}{$\begin{array}{l}\text { (6) } \\
\text { Aconselhamento } \\
\text { e atendimento } \\
\text { em saúde sexual } \\
\text { e reprodutiva } \\
\text { (SSR) }\end{array}$} & $\begin{array}{c}12-15 \\
+ \text { de } \\
15\end{array}$ & $\begin{array}{l}\text { O corpo humano } \\
\text { e o seu } \\
\text { desenvolvimento }\end{array}$ & $\begin{array}{l}\text { - Serviços onde os } \\
\text { adolescentes podem ir } \\
\text { quando tiverem problemas } \\
\text { relacionados com estes } \\
\text { assuntos. }\end{array}$ & \multirow[t]{2}{*}{$\mathrm{X}$} & \multirow[t]{2}{*}{$\mathrm{X}$} \\
\hline & & $\begin{array}{l}\text { Sexualidade, } \\
\text { saúde e bem- } \\
\text { estar }\end{array}$ & $\begin{array}{l}\text { - Sistemas de cuidados de } \\
\text { saúde e serviços. }\end{array}$ & & \\
\hline
\end{tabular}




\section{Dimensões e atribuição dos itens}

Este instrumento de autorresposta contempla, como já foi referido, alguns dos conteúdos de educação sexual previstos em meio escolar (Portaria n. $\left.{ }^{\circ} 196-\mathrm{A} / 2010 ; \mathrm{WHO}, 2010\right)$, referentes ao domínio da informação, especificamente organizados em seis áreas temáticas: (1) Primeira relação sexual e preocupações sexuais (itens 1, 3, 14, 17 e 22); (2) Sexualidade e prazer sexual (itens 6, 15 e 23); (3) Contraceção e práticas sexuais seguras (itens 7, 8, 9, 11, 19 e 26); (4) Prevenção da Gravidez (itens 2 e 13); (5) Infeções sexualmente transmissíveis e VIH/SIDA (itens 4, 5, 10, 12, 16, 18, 21 e 24); e (6) Aconselhamento e atendimento em saúde sexual e reprodutiva (SSR) (itens 20 e 25).

A escala de resposta é dicotómica, ou seja, verdadeiro/falso, o que permite a cotação de um (1) para respostas corretas (verdadeiro) e zero (0) para respostas incorretas (falso). Um (1) significa que a resposta é dada no sentido correto, indicando conhecimentos adequados sobre a sexualidade. Itens não respondidos são cotados como zero (0). Para evitar tendências de resposta, os itens foram construídos de forma a representarem conceitos positivos e negativos. Os itens 1, 3, 4, 6, 9, 10, 13, 14, 15, 16, 19, 21, 25 são pontuados inversamente e o resultado consiste no somatório das respostas corretas. As pontuações mais elevadas refletem níveis mais elevados de conhecimentos e os resultados possíveis desta versão final variam entre 0 e 26.

\section{Procedimentos}

A amostra foi recolhida em 7 escolas (6 públicas e 1 semiprivada) das regiões Centro, Lisboa e Vale do Tejo. A autorização ética foi obtida pela Comissão Nacional de Proteção de Dados e pela Direção-Geral de Educação do Ministério de Educação Português (inquérito n.0389000001). Todos os adolescentes participaram voluntariamente no preenchimento do protocolo de investigação na sala de aula, com autorização dos pais, na presença de um/a professor/a e da investigadora principal. A recolha de dados decorreu entre fevereiro de 2013 e março de 2014.

\section{Análise de Dados}

Para a análise dos dados foram utilizadas estatísticas descritivas e inferenciais, por meio do programa SPSS versão 20 (SPSS Inc, Chicago, IL). 
Para avaliar a qualidade dos itens foram estudadas duas caraterísticas fundamentais: o índice de dificuldade (ID) e o índice de discriminação (D). O índice de dificuldade (ID) de um item é dado pela proporção de sujeitos que acertam o item (Teoria Clássica dos Testes) (Muñiz, 2003), ou seja, quanto maior é o ID, menos difícil é o item. Neste sentido, um teste de conhecimentos deve ter um contínuo de ID que vá desde itens fáceis até itens difíceis. O índice de discriminação (D) consiste na capacidade do item de diferenciar os alunos de maior conhecimento daqueles com menor conhecimento, correspondendo à diferença entre a proporção de acerto do primeiro grupo e a do segundo grupo. Quando a distribuição das pontuações é aproximada da curva normal, são considerados $27 \%$ dos sujeitos com pontuações inferiores e $27 \%$ dos sujeitos com pontuações inferiores. O valor de D é a diferença na proporção de acertos entre os dois grupos e, por isso, uma maior diferença significa uma maior discriminação (D). Também procedemos à análise da correlação bisserial, que consiste numa medida de associação entre o desempenho no item e na pontuação total na prova e avalia a homogeneidade do item (Muñiz, 2003; Muñiz, Fidalgo, García-Cueto, Martínez, \& Moreno, 2005).

Foram verificados os pressupostos para o cálculo de estatística inferencial paramétrica, nomeadamente a distribuição das variáveis e a homocedasticidade. Não existiram pontuações outliers na amostra em estudo (Hair, Anderson, \& Tatham, 1995). Considerando a dimensão da amostra, aplica-se o Teorema do Limite Central (ou teorema de Lindberg-Levy), que afirma que para amostras grandes a distribuição tende para a normalidade (Durrett, 2010; Murteira, Ribeiro, Silva, \& Pimenta, 2001). A decisão foi reforçada pela análise dos histogramas e, por essa razão, optou-se por utilizar testes paramétricos (teste te teste ANOVA one-way). A homocedasticidade foi testada através do teste de Levene (Howell, 2013). Apesar de existir homogeneidade das variâncias, optámos pela prova robusta Brown-Forsythe, por existirem diferenças relevantes no tamanho dos grupos a comparar (Tabachnick, \& Fidell, 2007). Para as análises de correlação utilizou-se o coeficiente de correlação de Pearson (Howell, 2013). 


\section{Resultados}

\section{Análise dos itens pela Teoria Clássica de Testes: Índice de dificuldade e discriminação}

$\mathrm{Na}$ análise do índice de dificuldade, como se pode observar na tabela 1 , nenhum item foi tão fácil que tivesse obtido $100 \%$ de respostas corretas ou tão difícil que tivesse obtido $0 \%$ de respostas certas. No gráfico 1 verifica-se uma adequada progressão da dificuldade dos itens. Este resultado aponta no sentido de o teste ter uma representação adequada e ampla do constructo no que diz respeito à sua dificuldade.

Tabela 1 - Índice de dificuldade e índice de discriminação

\begin{tabular}{|c|c|c|c|c|c|c|}
\hline \multirow[b]{2}{*}{ Itens } & \multirow[b]{2}{*}{$\begin{array}{c}\text { Índice de } \\
\text { dificuldade }\end{array}$} & \multicolumn{5}{|c|}{ Discriminação } \\
\hline & & $\begin{array}{c}\% \text { acertos } \\
27 \% \text { superiores }\end{array}$ & $\begin{array}{c}\% \text { acertos } \\
27 \% \text { inferiores }\end{array}$ & D & $x^{2}$ & $\begin{array}{c}\text { Correlação } \\
\text { ponto-biserial }\end{array}$ \\
\hline 21 & 11.59 & 13.67 & 13.43 & 0.24 & $0.01^{\mathrm{ns}}$ & $.015^{\mathrm{ns}}$ \\
\hline 1 & 18.03 & 27.1 & 11.27 & 15.83 & $33.69^{\star \star \star}$ & $.167^{\star \star}$ \\
\hline 10 & 28.28 & 53.48 & 16.55 & 36.93 & $124.98^{\star \star \star}$ & $.312^{\star \star}$ \\
\hline 25 & 35.34 & 53.72 & 19.90 & 33.81 & $102.48^{\star \star}$ & $.274^{\star \star}$ \\
\hline 16 & 48.09 & 67.15 & 33.09 & 34.05 & $96.71^{\star \star}$ & $.281^{\star \star}$ \\
\hline 2 & 50.29 & 78.42 & 28.3 & 50.12 & $210.45^{\text {** }}$ & $.382^{\star \star}$ \\
\hline 14 & 52.36 & 76.02 & 35.73 & 40.29 & $137.26^{\star * *}$ & $.320^{\star *}$ \\
\hline 3 & 57.27 & 74.58 & 37.89 & 36.69 & $114.05^{\star \star *}$ & $.301^{\star \star}$ \\
\hline 18 & 62.14 & 80.34 & 43.17 & 37.17 & $121.96^{\star \star}$ & $.298^{\star \star}$ \\
\hline 11 & 62.20 & 87.29 & 36.93 & 50.36 & $224.69^{\star \star \star}$ & $.401^{\star \star}$ \\
\hline 13 & 63.11 & 79.86 & 46.04 & 33.81 & $102.21^{\text {k* }}$ & $.288^{\star \star}$ \\
\hline 12 & 69.32 & 88.73 & 50.12 & 38.61 & $146.42^{\star \star \star}$ & $.342^{\star \star}$ \\
\hline 9 & 74.00 & 95.2 & 48.2 & 47.00 & $227.02^{\star \star}$ & $.417^{\star \star}$ \\
\hline 6 & 76.33 & 94.24 & 50.6 & 43.65 & $198.86^{\star \star}$ & $.407^{\star \star}$ \\
\hline 26 & 76.57 & 89.45 & 67.15 & 22.3 & $61.03^{\star \star}$ & $.199^{\star \star}$ \\
\hline 22 & 77.99 & 89.45 & 65.71 & 23.74 & $67.56^{\star \star}$ & $.250^{\star \star}$ \\
\hline 15 & 79.09 & 97.36 & 52.04 & 45.32 & $226.63^{\text {«* }}$ & $.463^{\star \star}$ \\
\hline 4 & 79.64 & 88.73 & 67.39 & 21.34 & $55.45^{\star \star}$ & $.235^{\star \star}$ \\
\hline 8 & 80.08 & 96.88 & 56.35 & 40.53 & $191.16^{\star \star}$ & $.411^{\star \star}$ \\
\hline 19 & 80.65 & 97.36 & 50.6 & 46.76 & $236.86^{\star \star}$ & $.485^{\star \star}$ \\
\hline 20 & 87.31 & 96.88 & 71.94 & 24.94 & $98.56^{\star \star}$ & $.322^{\star *}$ \\
\hline 5 & 87.52 & 97.36 & 70.98 & 26.38 & $108.90^{\text {** }}$ & $.314^{\star \star}$ \\
\hline 24 & 90.61 & 94.24 & 84.65 & 9.59 & $20.33^{\star \star}$ & $.148^{\star \star}$ \\
\hline 17 & 92.62 & 99.76 & 82.73 & 17.03 & $75.68^{\star \star \star}$ & $.276^{\star \star}$ \\
\hline 23 & 92.88 & 97.84 & 86.09 & 11.75 & $38.97^{\star *}$ & $201^{\star \star}$ \\
\hline 7 & 98.25 & 99.04 & 95.44 & 3.60 & $10.06^{\star}$ & $.145^{\star \star}$ \\
\hline
\end{tabular}

Como se pode observar no gráfico 2 , as pontuações obtiveram uma distribuição aproximada à curva normal, variaram entre 6 e 25, com uma média de 17.32 ( $\mathrm{DP}=3.19$ ). Neste caso, e considerando que a curva observada no histograma (Gráfico 2) aproxima-se muito da curva normal, tornou-se possível avaliar a capacidade discriminativa (D) dos itens. Assim sendo, para a sua avaliação foram criados dois grupos com 417 sujeitos (considerando que $27 \%$ de 1545 são 417 sujeitos) cada, a que correspondem 
as pontuações mais altas e mais baixas. Na tabela 1 são apresentadas as percentagens de acerto para o grupo de desempenho superior e inferior.

\section{Gráfico 1 - Ordenação dos itens pelo Índice de Dificuldade}

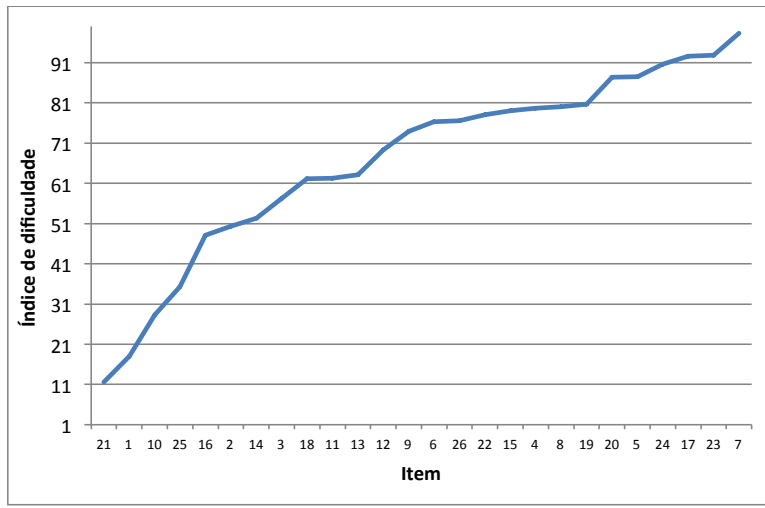

Gráfico 2 - Histograma das pontuações na escala de conhecimentos dos adolescentes

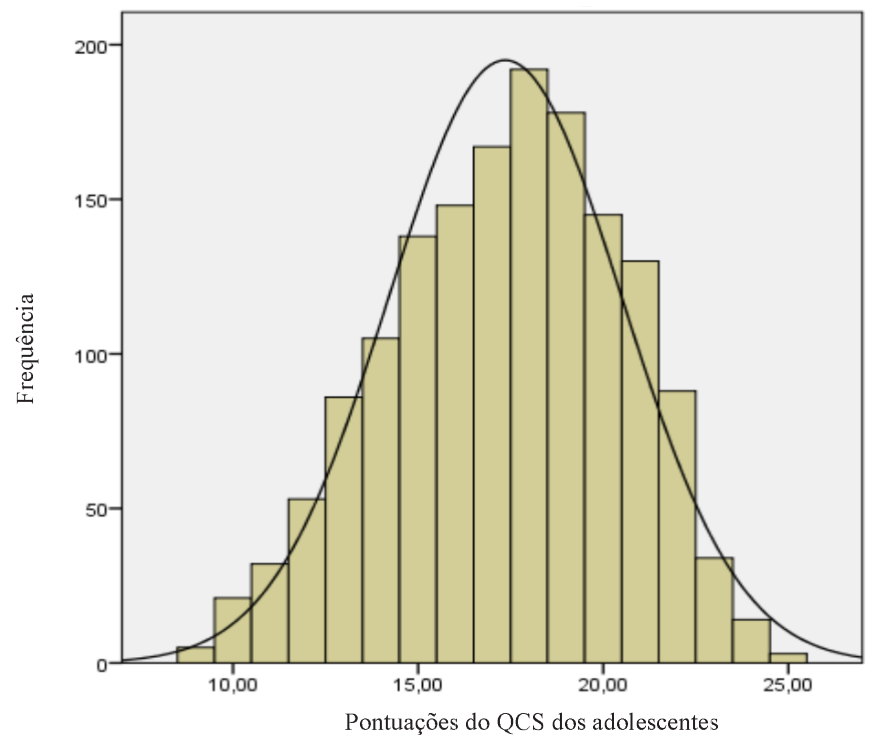


À estatística dos valores de discriminação (D) foi associado um teste de diferenças (Qui-quadrado, por se tratarem de duas variáveis dicotómicas). Apenas o item 21 não discriminou os grupos ( $\left.D=0.24 ; \chi^{2}(1)=0.01, p=.919\right)$. Naturalmente que os itens mais fáceis tendem a ser menos discriminativos, porque a maior parte da amostra os acerta; contudo, fazem parte do continuum que representa o grau de conhecimentos. A discriminação do item foi ainda avaliada através da correlação ponto-bisserial quando uma das variáveis é dicotómica e a outra intervalar (Santisban-Requenha, 1990). Mais uma vez, apenas o item 21 se mostrou desadequado (Correlação pontobiserial=.015) (cf. Tabela 1). Foi também calculada a homogeneidade do item, que sugere a exclusão do item 21 do total do teste.

\section{Análise de diferenças relacionadas com o género, idade, nível de escolaridade, religião, nacionalidade e área de residência (urbana versus rural)}

$\mathrm{Na}$ análise de diferenças, verifica-se que as raparigas apresentam médias ( $M=17.73$; $D P=3.09$ ) mais elevadas nos conhecimentos do que os rapazes $(M=16.92 ; \quad D P=3.25)$ e as diferenças observadas foram estatisticamente significativas [t(1543)=-4.982; $\mathrm{p}=.001$ ] (Tabela 2 e Tabela 3).

Os adolescentes com idades acima dos 15 anos (15-17 e 18-20) diferenciam-se do grupo que tem entre 12 a 14 anos nos conhecimentos em sexualidade, apresentando médias mais elevadas do que os jovens com idades inferiores aos 15 anos $[F(2,831.968)=84.578 ; p=.001]$. Da mesma forma, os alunos que frequentam o $9^{\circ}$ ano do Ensino Básico e o Ensino Secundário revelam médias superiores nos conhecimentos relativamente aos alunos que frequentam $07^{\circ}$ e $8^{\circ}$ anos de escolaridade $[F(2$, $1051.665)=214.554 ; p=.001]$. São os alunos entre os 18 e os 20 anos $(M=18.06$; $D P=3.06)$, que se encontram a frequentar $011^{\circ}$ e $12^{\circ}$ anos ( $M=18.48 ; \quad D P=2.83$ ), que evidenciam médias mais elevadas nos conhecimentos sobre sexualidade. Importa ainda salientar que a idade obteve uma correlação positiva e significativa com os conhecimentos dos adolescentes sobre sexualidade $(r=.328, p=.001)$, indicando que, à medida que os adolescentes crescem, vão tendo mais informação e sabem mais sobre a sexualidade, como também se pode verificar na tabela 3 . 
O grupo dos adolescentes que se considera religioso $(n=956)$ obteve valores inferiores nos conhecimentos sobre sexualidade $(M=17.11 ; D P=3.18)$ comparativamente com os adolescentes que referem não pertencer a nenhuma religião $(M=17.68 ; \quad D P=3.18)$ e as diferenças revelam-se estatisticamente significativas $[\mathrm{t}(1529)=-3.396 ; \mathrm{p}=.001]$. Foram os adolescentes portugueses que obtiveram médias superiores nos conhecimentos sobre a sexualidade $(M=17.39 ; \quad D P=3.18)$, quando comparados com os adolescentes de outras nacionalidades $(M=16.17$; $\mathrm{DP}=3.43)$, com diferenças significativas [t(1523) $=3.280 ; p=.001$ ]. Não se encontraram diferenças estatisticamente significativas nos conhecimentos dos adolescentes que vivem em meio urbano e em meio rural $[t(1523)=-1.31$; $\mathrm{p}=.067$ ] (Tabela 2).

Tabela 2 - Médias, desvios-padrão e testes de diferenças dos conhecimentos sobre sexualidade em função do género, idade, anos de escolaridade, religião, nacionalidade e área de residência

\begin{tabular}{|c|c|c|c|c|c|c|c|}
\hline $\begin{array}{l}\text { Conhecimentos } \\
\text { sobre sexualidade }\end{array}$ & $\mathbf{n}$ & M & DP & Teste & GI & $\mathbf{p}$ & Post-hoc \\
\hline \multicolumn{8}{|l|}{ Género } \\
\hline Masculino & 790 & 16.92 & 3.25 & \multirow[t]{2}{*}{$-4.982^{a}$} & \multirow[t]{2}{*}{1543} & \multirow[t]{2}{*}{.001} & \multirow{2}{*}{ - } \\
\hline Feminino & 755 & 17.73 & 3.09 & & & & \\
\hline \multicolumn{8}{|l|}{ Idade } \\
\hline $12-14$ anos & 224 & 14.92 & 3.09 & \multirow[t]{3}{*}{$84.578^{b}$} & \multirow[t]{3}{*}{2} & \multirow[t]{3}{*}{.001} & \multirow{3}{*}{$\begin{array}{l}12-14 \text { anos }<15-17 \text { e } \\
18-20 \text { anos }\end{array}$} \\
\hline $15-17$ anos & 879 & 17.59 & 3.03 & & & & \\
\hline $18-20$ anos & 417 & 18.06 & 3.06 & & & & \\
\hline \multicolumn{8}{|c|}{ Anos de escolaridade } \\
\hline $7^{\circ}$ e $8^{\circ}$ anos & 255 & 14.45 & 2.63 & \multirow[t]{3}{*}{$214.554^{b}$} & \multirow[t]{3}{*}{2} & \multirow[t]{3}{*}{.001} & \multirow{3}{*}{$\begin{array}{l}7^{\circ} \text { e } 8^{\circ}<9^{\circ} \text { e } 10^{\circ}, 11^{\circ} \\
\text { e } 12^{\circ}\end{array}$} \\
\hline $9^{\circ}$ e $10^{\circ}$ anos & 452 & 16.78 & 2.96 & & & & \\
\hline $11^{\circ} \mathrm{e} 12^{\circ}$ anos & 838 & 18.48 & 2.83 & & & & \\
\hline \multicolumn{8}{|l|}{ Religião } \\
\hline Sim & 965 & 17.11 & 3.18 & \multirow[t]{2}{*}{$-3.396^{a}$} & \multirow[t]{2}{*}{1529} & \multirow[t]{2}{*}{.001} & \multirow{2}{*}{ - } \\
\hline Não & 566 & 17.68 & 3.18 & & & & \\
\hline \multicolumn{8}{|l|}{ Nacionalidade } \\
\hline Portuguesa & 1447 & 17.39 & 3.18 & \multirow[t]{2}{*}{$3.280^{\mathrm{a}}$} & \multirow[t]{2}{*}{1523} & \multirow[t]{2}{*}{.001} & \multirow{2}{*}{-} \\
\hline Outra & 78 & 16.17 & 3.43 & & & & \\
\hline \multicolumn{8}{|l|}{ Área de residência } \\
\hline Urbana & 483 & 17.55 & 3.11 & \multirow[t]{2}{*}{$-1.831^{a}$} & \multirow[t]{2}{*}{1523} & \multirow[t]{2}{*}{.067} & \multirow{2}{*}{-} \\
\hline Rural & 1042 & 17.22 & 3.23 & & & & \\
\hline
\end{tabular}


Tabela 3 - Médias e desvios-padrão dos conhecimentos por género e ano de escolaridade

\begin{tabular}{cccc}
\hline \multirow{2}{*}{ Ano de escolaridade } & \multicolumn{2}{c}{ Género } & Total \\
& Masculino & Feminino & Média (DP) \\
& Média (DP) & Média (DP) & $14.01(2.64)$ \\
$7^{\circ}$ ano & $13.7(2.52)$ & $14.4(2.76)$ & $14.86(2.56)$ \\
$8^{\circ}$ ano & $14.7(2.59)$ & $15.1(2.52)$ & $16.23(3.26)$ \\
$9^{\circ}$ ano & $15.5(3.12)$ & $17.6(3.14)$ & $16.96(2.84)$ \\
$10^{\circ}$ ano & $16.7(3.12)$ & $17.2(2.59)$ & $17.87(2.68)$ \\
$11^{\circ}$ ano & $17.7(2.82)$ & $17.9(2.56)$ & $18.77(2.85)$ \\
$12^{\circ}$ ano & $18.4(2.79)$ & $19.2(2.86)$ & \\
\hline
\end{tabular}

\section{Análises de diferenças relacionadas com a educação sexual familiar e escolar}

Relativamente à educação sexual obtida em contexto familiar, verificouse que os adolescentes que tiveram a mãe como educadora sexual apresentam médias mais elevadas nos conhecimentos sobre a sexualidade $(\mathrm{M}=17.47$; $\mathrm{DP}=3.21)$ do que os adolescentes que tiveram o pai a desempenhar a mesma função $(M=16.84 ; D P=3.23)$, com diferenças estatisticamente significativas $[F(2,618.886)=3.276 ; p=.038]$. Os adolescentes cujas mães têm formação superior apresentam valores mais elevados nos conhecimentos sobre a sexualidade do que o grupo de adolescentes em que as mães têm o $1^{\circ}$ ciclo do ensino básico $[F(4,1253.359)=3.351 ; p=.010]$. Não foram encontradas diferenças estatisticamente significativas nos conhecimentos dos adolescentes sobre sexualidade no que respeita à escolaridade do pai $[F(4$, 1274.883)=.872; $p=.480$ ]. Os adolescentes com mais conhecimentos sobre a sexualidade consideraram que a educação sexual recebida em casa, em função do que precisavam, foi insuficiente (fraca) $(M=18.48, D P=3.00)$, apresentando diferenças significativas $[F(3,769.236)=7.161 ; p=.001]$, quando comparados com os adolescentes que consideram ter recebido uma muito boa $(M=17.02$; $D P=3.16)$ e boa $(M=17.51$; $D P=3.25)$ educação sexual, e mesmo com os que dizem não ter recebido ( $M=17.31$; $D P=3.18)$.

Relativamente à educação sexual recebida na escola, verifica-se que o grupo que não participou em nenhuma atividade relacionada com a sexualidade apresentou médias inferiores nos conhecimentos ( $M=16.65$; $D P=3.77)$, quando comparado com os adolescentes que tiveram mais de 5 anos de educação sexual (5-6 e + 7 anos) $(M=18.45 ; \quad D P=2.97$ e $M=18.99 ; \quad D P=2.80$ respetivamente). Por outro lado, também se encontraram diferenças 
estatisticamente significativas nos conhecimentos entre os adolescentes que tiveram até 2 anos de educação sexual na escola $(M=16.83$; $D P=3.26)$ e os que tiveram mais de 3 anos, apresentando estes últimos médias mais elevadas. 0 grupo de adolescentes que teve entre 5 e 6 anos e mais de 7 anos de educação sexual na escola obteve valores mais elevados nos conhecimentos $(M=18.45$, $\mathrm{DP}=2.97 ; \mathrm{M}=18.99, \mathrm{DP}=2.80$ respetivamente) do que o grupo de adolescentes que teve 3 a 4 anos de educação sexual em meio escolar $(M=17.67 ; D P=3.05)$ $[F(4,319.843)=16.267 ; p=.001]$. Os adolescentes com mais conhecimento em sexualidade consideram que a educação sexual recebida na escola foi insuficiente (fraca/má) $(\mathrm{M}=17.94 ; \mathrm{DP}=3.30)$ e diferenciam-se dos adolescentes que consideraram que a educação sexual recebida na escola foi de muito boa qualidade $(\mathrm{M}=16.95 ; \mathrm{DP}=3.26)$, apresentando diferenças estatisticamente significativas $[F(4,579.205)=2.667 ; p=.032]$ (Tabela 4).

Tabela 4 - Médias, desvios-padrão e testes de diferenças dos conhecimentos sobre sexualidade em função da educação sexual recebida em casa e na escola

\begin{tabular}{|c|c|c|c|c|c|c|c|}
\hline $\begin{array}{l}\text { Conhecimentos sobre } \\
\text { sexualidade }\end{array}$ & $\mathbf{n}$ & M & DP & Teste $^{a}$ & gl & $\mathbf{p}$ & Post-hoc \\
\hline \multicolumn{8}{|c|}{ Educação Sexual na Família } \\
\hline \multicolumn{8}{|c|}{ Pessoa responsável pela ES em casa } \\
\hline Pai & 199 & 16.84 & 3.23 & \multirow[t]{3}{*}{3.276} & \multirow[t]{3}{*}{2} & \multirow[t]{3}{*}{.038} & \multirow[t]{3}{*}{ Pai < Mãe } \\
\hline Mãe & 948 & 17.47 & 3.21 & & & & \\
\hline Não tive & 257 & 17.26 & 3.11 & & & & \\
\hline \multicolumn{8}{|l|}{ Escolaridade do pai } \\
\hline $1^{\circ} \mathrm{Ciclo}$ & 287 & 17.17 & 3.29 & \multirow[t]{5}{*}{.872} & \multirow[t]{5}{*}{4} & \multirow[t]{5}{*}{.480} & \multirow[t]{5}{*}{-} \\
\hline $2^{\circ}$ Ciclo & 313 & 17.26 & 3.08 & & & & \\
\hline $3^{\circ}$ Ciclo & 376 & 17.39 & 3.09 & & & & \\
\hline Secundário & 321 & 17.40 & 3.26 & & & & \\
\hline Ensino Superior & 167 & 17.72 & 3.23 & & & & \\
\hline \multicolumn{8}{|l|}{ Escolaridade da mãe } \\
\hline $1^{\circ} \mathrm{Ciclo}$ & 201 & 16.91 & 3.08 & \multirow[t]{5}{*}{3.351} & \multirow[t]{5}{*}{4} & \multirow[t]{5}{*}{.010} & \multirow{5}{*}{$\begin{array}{l}1^{\circ} \text { Ciclo }<\text { Ensino } \\
\text { Superior }\end{array}$} \\
\hline $2^{\circ}$ Ciclo & 211 & 17.14 & 3.29 & & & & \\
\hline $3^{\circ}$ Ciclo & 461 & 17.42 & 3.16 & & & & \\
\hline Secundário & 410 & 17.36 & 3.20 & & & & \\
\hline Ensino Superior & 230 & 17.96 & 3.07 & & & & \\
\hline \multicolumn{8}{|c|}{ ES recebida em casa em função do que precisava } \\
\hline Muito boa & 675 & 17.02 & 3.16 & \multirow[t]{4}{*}{7.161} & \multirow[t]{4}{*}{3} & \multirow[t]{4}{*}{.001} & \multirow{4}{*}{$\begin{array}{l}\text { Muito boa }< \\
\text { Boa; Todos os } \\
\text { grupos < Fraca }\end{array}$} \\
\hline Boa & 498 & 17.51 & 3.25 & & & & \\
\hline Fraca/má & 101 & 18.48 & 3.00 & & & & \\
\hline Não recebeu & 257 & 17.31 & 3.18 & & & & \\
\hline \multicolumn{8}{|c|}{ Educação Sexual na escola } \\
\hline \multicolumn{8}{|l|}{ Anos de ES na escola } \\
\hline Não teve ES & 49 & 16.65 & 3.77 & \multirow[t]{5}{*}{16.267} & 4 & \multirow[t]{5}{*}{.001} & \multirow{5}{*}{ 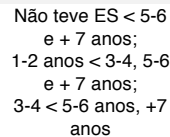 } \\
\hline $1-2$ anos & 701 & 16.83 & 3.26 & & & & \\
\hline 3-4 anos & 443 & 17.67 & 3.05 & & & & \\
\hline $5-6$ anos & 187 & 18.45 & 2.97 & & & & \\
\hline+7 anos & 68 & 18.99 & 2.80 & & & & \\
\hline \multicolumn{8}{|c|}{ Qualidade da ES recebida na escola } \\
\hline Muito boa & 296 & 16.95 & 3.26 & 2.667 & 4 & .032 & Muito boa $<$ \\
\hline Boa & 583 & 17.31 & 3.15 & & & & Fraca/má \\
\hline Suficiente & 446 & 17.42 & 3.10 & & & & \\
\hline Fraca/má & 160 & 17.94 & 3.30 & & & & \\
\hline Não tive ES & 56 & 17.33 & 3.30 & & & & \\
\hline
\end{tabular}


Análise de correlações com as atitudes face ao preservativo e de diferenças no uso do preservativo na última relação sexual

O nível de conhecimentos sobre a sexualidade mostrou-se associado com algumas dimensões da Escala Multidimensional de Atitudes em Relação à Utilização do Preservativo (EMARUP) (Neto, 2004). As correlações são positivas, o que parece indicar que quanto maior é o conhecimento dos adolescentes sobre a sexualidade, mais positivas são as suas atitudes face ao preservativo. Os resultados evidenciam que os conhecimentos sobre a sexualidade se correlacionam de forma significativa, embora pouco expressiva, com as atitudes favoráveis à fiabilidade e eficácia do preservativo ( $r=.268$, $p=.001$ ) e moderadamente (Cohen, 1988) com a negociação ( $r=.358, p=.001)$ da utilização do preservativo e com a imagem social positiva dos utilizadores $(r=.403, p=.001)$ do preservativo. Entre os resultados, importa ainda salientar que os adolescentes que se sentem confortáveis na negociação do preservativo com o par amoroso apresentam, como seria de esperar, mais conforto na compra do preservativo $(r=.351, p=.001)$, e os que valorizam os pares que usam preservativo apresentam mais conforto nos momentos de negociação do preservativo com o/a parceiro/a ( $r=.587, p=.001$ ) (Quadro 2).

Os adolescentes que referiram ter usado preservativo no último encontro sexual apresentam médias mais elevadas $(M=17.89$; $D P=3.08)$ nos conhecimentos sobre sexualidade do que os adolescentes que não usaram preservativo $(M=17.26 ; D P=3.25)$ e essa diferença é estatisticamente significativa $[t(808)=2.695 ; p=.007]$.

\section{Quadro 2 - Correlações entre os conhecimentos sobre a sexualidade e as dimensões da Escala Multidimensional de Atitudes em Relação à Utilização do Preservativo (EMARUP)}

\begin{tabular}{|c|c|c|c|c|c|c|}
\hline & $\begin{array}{l}\text { Conhe- } \\
\text { cimentos }\end{array}$ & Fiabilidade & $\begin{array}{l}\text { Prazer } \\
\text { Sexual }\end{array}$ & $\begin{array}{l}\text { Embaraço } \\
\text { na compra }\end{array}$ & $\begin{array}{c}\text { Embaraço } \\
\text { na } \\
\text { negociação }\end{array}$ & $\begin{array}{c}\text { Imagem } \\
\text { Social } \\
\text { negativa }\end{array}$ \\
\hline Conhecimentos & 1 & & & & & \\
\hline Fiabilidade & $.268^{\star \star}$ & 1 & & & & \\
\hline Prazer sexual & $.098^{\star \star}$ & $.179^{\star \star}$ & 1 & & & \\
\hline $\begin{array}{l}\text { Embaraço na } \\
\text { compra }\end{array}$ & $.081^{\star \star}$ & $.060^{*}$ & .014 & 1 & & \\
\hline $\begin{array}{l}\text { Embaraço na } \\
\text { negociação }\end{array}$ & $.358^{\star *}$ & $.314^{\star \star}$ & $.080^{\star \star}$ & $.351^{\star *}$ & 1 & \\
\hline Imagem Social & $.403^{\star \star}$ & $.319^{\star \star}$ & $.221^{\star \star *}$ & $.181^{\star \star}$ & $.587^{\star \star \star}$ & 1 \\
\hline
\end{tabular}




\section{Conclusões}

O Questionário de Conhecimentos sobre Sexualidade (QCS) mostrouse adequado, tendo-se verificado que a operacionalização do constructo revelou relevância e amplitude teórica, para o que foi determinante o contributo dado pelos júris. Este instrumento revela-se apropriado para o diagnóstico de necessidades de formação, uma vez que avalia os conhecimentos num contínuo de dificuldade, com itens fáceis e difíceis, o que permite distinguir o grupo dos alunos com mais e menos conhecimento. Por meio dos índices de dificuldade e discriminação, é possível afirmar que o QCS é composto por um conjunto de itens de boa qualidade na avaliação dos conhecimentos dos adolescentes sobre os temas que compõem o instrumento. A versão final do QCS é constituída por 25 itens, distribuídos em 6 temáticas: (1) Primeira relação sexual e preocupações sexuais; (2) Sexualidade e prazer sexual; (3) Contraceção e práticas sexuais seguras; (4) Prevenção da gravidez; (5) Infeções sexualmente transmissíveis e VIH/SIDA; e (6) Aconselhamento e atendimento em saúde sexual e reprodutiva (SSR).

À semelhança dos resultados obtidos nos estudos de Matos et al. (2013) e de Vilar e Ferreira (2009), os dados obtidos com o QCS apontam no sentido de que as raparigas têm mais informação e conhecimento sobre a sexualidade do que os rapazes, o que requer maior atenção dos programas de ES nas escolas em relação ao género masculino. Considerando os resultados do estudo de Matos, Simões, Camacho, Reis, e Equipa da Aventura Social (2015), que ilustram o início da atividade sexual em alguns grupos de jovens antes dos 15 anos, os resultados obtidos neste estudo apontam no sentido de estes jovens estarem mais vulneráveis a comportamentos sexuais de risco. Este aspeto contribui para reforçar a necessidade da ES começar antes de os adolescentes iniciarem as relações sexuais. Os adolescentes com mais de 5 anos de educação sexual na escola e, principalmente, os que tiveram 7 ou mais anos de educação sexual são os que revelam mais conhecimentos sobre a sexualidade. São os adolescentes mais velhos que têm mais formação e anos de educação sexual, o que muito, certamente, se deve ao impacto da educação sexual em contexto escolar. Por outro lado, jovens que não participaram em atividades relacionadas com esta área prioritária de intervenção em meio escolar foram os que revelaram ter menos informação e conhecimento sobre sexualidade, tal como é referido na 
literatura (Ramiro, Reis, Matos, \& Dinis, 2011; Vilar \& Ferreira, 2009). Neste sentido, a educação sexual em meio escolar deve começar o mais cedo possível, de preferência desde o pré-escolar.

Neste estudo, os adolescentes de nacionalidade portuguesa e que não pertencem a nenhuma religião foram os que obtiveram valores mais elevados nos conhecimentos sobre os temas da sexualidade do questionário. Estes dados vão ao encontro da literatura (Matos, 2008) e contribuem para reforçar a importância de conhecer a realidade dos jovens de diversas nacionalidades, que vivem em Portugal, de modo a identificar caraterísticas, necessidades e fatores ligados ao risco e à proteção, para definir áreas de intervenção de acordo com as especificidades culturais desses grupos (Matos, 2008). Tornase fundamental desenvolver políticas de promoção da saúde nestas comunidades, de modo a integrar os alunos de diversas nacionalidades e culturas, através de uma abordagem multicultural assente no estabelecimento de estratégias preventivas e de promoção da saúde sexual e reprodutiva, à qual todos devem ter acesso e da qual todos devem beneficiar.

O facto de os adolescentes com mais conhecimentos terem tido a mãe como educadora sexual, quando comparados com os adolescentes que tiveram o pai a desempenhar a mesma função, não é surpreendente. A literatura da área indica que são as mães que estão mais disponíveis e que mais conversam com os filhos sobre a sexualidade (Matos et al., 2010). Neste sentido, importa envolver os progenitores do género masculino em formação parental, capacitando-os para uma melhor comunicação com os filhos sobre educação sexual, já que estes podem ter um papel importante junto dos rapazes (Matos et al., 2010). O nível de escolaridade das mães foi também uma variável que revelou ter impacto na informação e conhecimento dos filhos sobre sexualidade, o que reforça a necessidade de atuar junto de famílias cujas mães tenham pouca ou nenhuma escolaridade.

Os adolescentes com maior nível de informação parecem ser também mais críticos e conscientes de que a ES recebida em casa e na escola fora insuficiente, considerando as suas necessidades. Estes dados confirmam, mais uma vez, que a ES deve ser feita em função do que os jovens precisam, e não tanto em função do que os adultos consideram ser as necessidades formativas dos adolescentes. Aumentar os canais de comunicação e assegurar mecanismos de identificação das necessidades dos adolescentes 
sobre saúde sexual e reprodutiva pode revelar-se mais eficaz em matéria de prevenção.

Embora seja unânime entre a comunidade científica que o conhecimento, por si só, não é suficiente para a modificação dos comportamentos (Fisher \& Fisher, 1992; Kalichman et al., 2002), este pode exercer influência nas atitudes e, consequentemente, contribuir para diminuir comportamentos de risco. Os resultados deste estudo apontam para a existência de associações entre os conhecimentos e as atitudes face ao preservativo, corroborando os achados da literatura (Reis \& Matos, 2007; Roque, 2001; Synovitz et al., 2002). Foi possível, então, verificar que os conhecimentos dos adolescentes parecem ter influência positiva nas atitudes favoráveis relacionadas com a fiabilidade e eficácia do preservativo, com o conforto nos momentos de negociação do preservativo com o/a parceiro/a e, ainda, com a valorização dos pares/amigos que usam preservativo. Foi igualmente possível observar que os adolescentes que utilizaram preservativo no último encontro sexual foram os que revelaram estar mais informados sobre a sexualidade.

Estes dados permitem reforçar a importância de garantir que os adolescentes possam esclarecer as suas dúvidas e tenham acesso a informação correta, atual e completa, atendendo às suas reais necessidades, seja em contexto familiar ou escolar. A literacia em saúde aplicada ao problema do VIH/SIDA em populações jovens é cada vez mais necessária para o desenvolvimento de um conjunto de competências cognitivas e sociais, consideradas imprescindíveis para aceder, compreender e usar a informação (WHO, 2013), a fim de melhorar a sua saúde sexual. O QCS poderá ser um instrumento de utilidade para as escolas e organizações que pretendam planear intervenções de prevenção dos comportamentos sexuais de risco e de promoção de comportamentos preventivos.

\section{Notas}

1 Este artigo foi realizado no âmbito de uma investigação de Doutoramento em Ciências da Educação, financiada pela Fundação para a Ciência e a Tecnologia FCT (Referência SFRH/BD/75130/2010). 
2 A palavra 'adolescência' vem do latim adolescere, que significa "crescer", apontando para um período de mudanças (Silva, 2004), enquanto fase de desenvolvimento com caraterísticas específicas (Medeiros, 2000). Para a Organização Mundial de Saúde, a adolescência compreende o período entre os 11 e os 19 anos de idade, desencadeado por mudanças corporais e fisiológicas. De modo geral, a adolescência é considerada um espaço de transição entre a infância e a idade adulta, marcada por alterações biopsicossociais (Silva, 2004). Alguns autores defendem a existência de tantas adolescências quanto adolescentes, uma vez que a forma como se concretiza em cada adolescente pode ser diferente (Silva, 2004).

\section{Referências}

Carvalho, C., \& Pinheiro, M. P. (2013). Educação familiar e comunicação sobre sexualidade: As necessidades de (in)formação de pais e filhos. In A. Pereira, M. M. Calheiros, P. Vagos, I. Direito, S. Monteiro, \& A. Allan Gomes (Orgs.), Atas do VIII Simpósio Nacional de Investigação em Psicologia (pp. 138-147). Aveiro: Associação Portuguesa de Psicologia.

Cohen, J. (1988). Statistical power analysis for the behavioral sciences. Hillsdale, NJ: Lawrence Erlbaum Associates.

Degroote, S., Vogelaers, D., Liefhooghe, G., Vermeir, P., \& Vandijck, D. (2014). Sexual experience and HIV-related knowledge among Belgian university students: A questionnaire study. BMC Research Notes, 7(299). doi:10.1186/1756-0500-7299

Durrett, R. (2010). Probability: Theory and examples (4th ed., Cambridge Series in Statistical and Probabilistic Mathematics). Cambridge University Press: Cambridge.

Farih, M., Khan, K., Freeth, D., \& Meads, C. (2014). Protocol study: Sexual and reproductive health knowledge, information-seeking behaviour and attitudes among Saudi women: A questionnaire survey of university students. Reproductive Health, 11(34). doi:10.1186/1742-4755-11-34

Fisher, J., \& Fisher, W. (1992). Changing AIDS risk behavior. Psychological Bulletin, 111, 455-474.

Hair, J., Anderson, R. E., \& Tatham, R. L. (1995). Multivariate data: Analysis with readings. New Jersey: Prentice-Hall.

Howell, D. (2013). Statistical methods for psychology (8th ed.). Duxbury: Pacific Grove.

International Planned Parenthood Federation (2007). O projeto SAFE. Um guia para o desenvolvimento de políticas sobre direitos e saúde sexual e reprodutiva de jovens na Europa. Lisboa: APF - Associação para o Planeamento Familiar.

International Planned Parenthood Federation (2008). Sexual rights: An IPPF declaration. London: IPPF.

Kalichman, S., Stein, J., Malow, R., Averhart, C., Dévieux, J., Jennings, T., \& Feaster, J. (2002). Predicting protected sexual behaviour using the Information-Motivation- 
Behaviour skills model among adolescent substance abusers in court-ordered treatment. Psychology, Health \& Medicine, 7(3), 327-338. doi: 10.1080/ 13548500220139368

Kirby, D., Laris, B. A., \& Rolleri, L. (2007). Sex and HIV education programs: Their impact on sexual behaviors of young people throughout the world. Journal of Adolescent Health, 40, 206-217.

Martins, M. (2010). Conhecimentos e comportamentos sobre algumas infecções sexualmente transmissíveis dos alunos dos ensinos básico e secundário de uma escola da área da grande lisboa (Tese de Mestrado não publicada). Universidade Nova de Lisboa / Instituto da Higiene e Medicina Tropical, Lisboa.

Matos, M. G. (Ed.). (2008). Sexualidade, segurança e SIDA - Estado da arte e propostas em meio escolar. Lisboa: IHMT/FMH/FCT.

Matos, M. G., Ramiro, L., Reis, M., \& Equipa Aventura Social (2013). Sexualidade dos jovens portugueses: Relatório do estudo online sobre sexualidade nos jovens (Online Study of Young People's Sexuality (OSYS) - Dados de 2011). Lisboa: Centro de Malária e Outras Doenças Tropicais/ IHMT/UNL/FMH/ Universidade Técnica de Lisboa.

Matos, M. G., Reis, M., Ramiro, L., \& Equipa Aventura Social (2011). Saúde sexual e reprodutiva dos estudantes do ensino superior: Relatório do estudo - Dados nacionais 2010. Lisboa: FMH/UTL /CMDT/IHMT/UNL.

Matos, M. G., Simões, C., Camacho, I., Reis, M., \& Equipa Aventura Social (2015). Relatório do estudo HBSC 2014 - A saúde dos adolescentes portugueses em tempos de recessão - Dados nacionais do estudo HBSC de 2014. Lisboa: Centro de Malária e Outras Doenças Tropicais/IHMT/UNL.

Matos, M., Simões, C., Tomé, G., Camacho, I., Ferreira, M., Ramiro, L., \& Equipa Aventura Social (2010). A saúde dos adolescentes portugueses - Relatório do estudo HBSC 2010. Lisboa: Centro de Malária e Outras Doenças Tropicais.

Medeiros, M. T. (2000). Conceito de adolescência. In M. T. Medeiros \& A. I. Serpa (Org.), Adolescência: Abordagens, investigações e contextos de desenvolvimento (pp. 12-27). Lisboa: Direcção Regional da Educação.

Ministério da Educação. (2000). A educação sexual em meio escolar. Linhas orientadoras. Lisboa: Ministério da Educação.

Muñiz, J. (2003). Teoría clásica de los tests. Madrid: Pirámide.

Muñiz, J., Fidalgo, A. M., García-Cueto, E., Martínez, R. J. \& Moreno, R. (2005). Análisis de los ítems. Madrid: La Muralla.

Murteira, B., Ribeiro, C., Silva, J., \& Pimenta, C. (2001). Introdução à estatística. Lisboa: Mc Graw-Hill.

Neto, F. (2004). Escala multidimensional de atitudes em relação à utilização do preservativo. Psychologica, 36, 219-234.

Paulos, L., \& Valadas, S. (2015). Avaliação da implementação da educação sexual em contexto escolar, na região do Algarve - A perspetiva de atores educativos responsáveis pela educação para a saúde/educação sexual. Revista Portuguesa de Educação, 28(2), 155-181. 
Ramiro, L. (2013). A educação sexual na mudança de conhecimentos, atitudes e comportamentos sexuais dos adolescentes (Dissertação de Doutoramento). Universidade Técnica de Lisboa/Faculdade de Motricidade Humana, Lisboa.

Ramiro, L., Reis, M., Matos, M., \& Diniz, J. (2011). Sex education among Portuguese adolescent students. Procedia - Social and Behavioral Sciences, 29, 493-502.

Ramiro, L., Reis, M., Matos, M., Diniz, J., \& Simões, C. (2011). Educação sexual, conhecimentos, crenças, atitudes e comportamentos nos adolescentes. Revista Portuguesa de Saúde Pública, 29(1), 11-21.

Reis, M., \& Matos, M. (2007). Conhecimentos e atitudes face ao uso de métodos contraceptivos e à prevenção das ISTs em jovens. Revista Lusófona de Ciências e Tecnologia da Saúde, 4(1), 23-35.

Reis, M., Ramiro, L., Matos, M., \& Diniz, J. (2013). Determinants influencing male condom use among university students in Portugal. International Journal of Sexual Health. 25(2), 115-127. doi: 10.1080/19317611.2012.728554

Roque, O. (2001). Semiótica da cegonha: Jovens, sexualidade e gravidez não desejada. Lisboa: Associação para o Planeamento da Família.

Sánchez, F. L. (1990). Educación sexual. Madrid: Fundación Universidad Empresa.

Santisban-Requenha, C. (1990). Psicometría: Teoría y práctica en la construcción de tests. Madrid: Ediciones Norma, S. A.

Silva, A. (2004). Desenvolvimento de competências sociais nos adolescentes. Perspectiva de prevenção em saúde mental na adolescência. Lisboa: Climepsi Editores.

Synovitz, L., Herbert, E., Kelley, R., \& Carlson, G. (2002). Sexual knowledge of college students in a southern State: Relationship to sexuality education results of Louisiana college student study shows need for sexuality programs. American Journal of Health Studies, 17, 163-172.

Tabachnick, B., \& Fidell, L. (2007). Using multivariate analysis (5th ed.). Needham Heights, MA: Allyn \& Bacon.

UNESCO. (2010). Orientação técnica internacional sobre educação sexual: Uma abordagem baseada em evidências para escolas, professores e educadores em saúde (Vol. 1 - Razões em favor da educação em sexualidade). Paris: UNESCO.

Vilar, D., \& Ferreira, P. (2009). A educação sexual dos jovens portugueses Conhecimentos e fontes. Educação Sexual em Rede, 5, 2-53.

World Health Organization. (2010). WHO Regional Office for Europe and BZgA Standards for sexuality education in Europe: A framework for policy makers, educational and health authorities and specialists. Cologne: Federal Centre for Health Education.

World Health Organization. (2013). Health literacy - The solid facts. Copenhagen: WHO/Regional Office for Europe. 


\section{Documentos legais}

Lei n. ${ }^{\circ} 60 / 2009$. Diário da República, $1 .^{a}$ série, N. ${ }^{\circ} 151$ A, de 6 de agosto. Estabelece o regime de aplicação da educação sexual em meio escolar.

Portaria n. ${ }^{\circ}$ 196-A/2010. Diário da República, $1 .^{a}$ série, $N{ }^{\circ} 69$, de 9 de abril. Regulamenta a Lei $n .^{\circ} 60 / 2009$, de 6 de agosto, que estabelece o regime de aplicação da educação sexual em meio escolar. 


\title{
KNOWLEDGE ABOUT SEXUALITY: CONSTRUCTION AND VALIDATION OF AN ASSESSMENT INSTRUMENT FOR ADOLESCENTS
}

\begin{abstract}
Knowledge, as a factor that exerts influence on attitudes, is a fundamental prerequisite in prevention programs of risky sexual behaviours. The aim of this study was to develop an instrument that would assess the knowledge that adolescents have about sexuality. The Knowledge about Sexuality Questionnaire assesses the knowledge in a difficulty continuum and also the distinction of students with different levels of knowledge. The sample was composed by 1545 adolescents with a mean age of 16 years $(S D=1.80)$ that attend school. The results show that girls are more informed than boys, and that Portuguese adolescents older than 15, without religion, with more than 5 years of sexual education in school and having their mothers as sexual educators are the ones with more knowledge about sexuality. The attitudes of the adolescents towards the use of condom are associated with the knowledge they have about it and the adolescents that used condom in their last sexual relation reveal higher levels of knowledge.
\end{abstract}

Keywords

Knowledge; Sexual Education; Adolescents; Questionnaire

\section{CONNAISSANCES DE LA SEXUALITÉ : LA CONSTRUCTION ET LA VALIDATION D'UN INSTRUMENT D'ÉVALUATION POUR LES ADOLESCENTS}

\section{Résumé}

En tant que potentiel facteur d'influence sur les attitudes, la connaissance est un préalable fondamental dans les programmes de préventions des comportements sexuels à risque. L'étude présentée ici a pour objectif de développer un instrument permettant d'évaluer les connaissances des adolescents sur la sexualité. Le Questionnaire des Connaissances sur la 
Sexualité permet ainsi d'évaluer les connaissances dans un continuum de difficultés et permet en outre de classer les élèves selon différents niveaux de savoir. L'échantillon utilisé est constitué de 1545 adolescents fréquentant des classes allant de la 5ème à la terminale $(M=16,44$ ans ; $D P=1,80)$. Les résultats ont permis de mettre en évidence que les filles sont plus informées que les garçons, et que les jeunes de plus de 15 ans, sans religion, ayant fréquenté les cours d'éducation sexuelle pendant plus de 5 ans et qui ont eu leur mère comme éducatrice sur les questions de sexualité sont les plus informés sur ce sujet. Les attitudes des adolescents au sujet du préservatif sont associées aux connaissances et ceux qui ont utilisé un préservatif lors de leur dernière relation sexuelle montre des niveaux plus élevés de connaissance.

Mots-clé

Connaissances; Éducation Sexuelle; Adolescents; Questionnaire

Recebido em maio 2016

Aceite para publicação em setembro 2017

i Centro de Investigação do Núcleo de Estudos e Intervenção Cognitivo-Comportamental (CINEICC), Faculdade de Psicologia e de Ciências da Educação da Universidade de Coimbra, Portugal.

ii Centro de Investigação do Núcleo de Estudos e Intervenção Cognitivo-Comportamental (CINEICC), Faculdade de Psicologia e de Ciências da Educação da Universidade de Coimbra, Portugal.

iii Centro de Investigação do Núcleo de Estudos e Intervenção Cognitivo-Comportamental (CINEICC), Faculdade de Psicologia e de Ciências da Educação da Universidade de Coimbra, Portugal.

iv Centro Lusíada de Investigação em Serviço Social e Investigação Social (CLISSIS), Universidade Lusíada de Lisboa, Portugal.

Toda a correspondência relativa a este artigo deve ser enviada para: Cristiana Pereira de Carvalho, Rua do Colégio Novo, 3000-115 Coimbra. E-mail: cristianapc@ hotmail.com 


\section{Anexo}

\section{Questionário de Conhecimentos sobre Sexualidade (QCS)}

1. Quase todos os jovens têm relações sexuais antes dos 18 anos.

2. Uma rapariga pode ficar grávida mesmo que o rapaz não ejacule dentro da vagina.

3. Depois da excitação e com o pénis em ereção, o homem deve ejacular porque podem surgir problemas se não o fizer.

4. A Sida pode apanhar-se através do beijo na boca.

5. O sexo oral e o sexo anal não possibilitam uma gravidez, mas podem provocar algumas doenças sexualmente transmissiveis.

6. A satisfação sexual não pode ser atingida sem penetração.

7. Antes da colocação do preservativo deve-se verificar sempre o estado de conservação da embalagem, a validade e o controlo de qualidade.

8. A pílula do dia seguinte só deverá ser utilizada como método de exceção e nunca regularmente.

9. Não existe risco de gravidez quando se utiliza o método "coito interrompido".

10. Uma pessoa que tem um teste VIH positivo, tem sida.

11. O consumo de álcool diminui a perceção dos riscos nos comportamentos sexuais.

12. Uma mulher pode apanhar o VIH se tiver sexo anal com um homem.

13. Uma rapariga não engravida se tiver tido relações sexuais durante a menstruação.

14. Ter sexo mantém uma relação amorosa.

15. A sexualidade restringe-se às relações sexuais.

16. Fazer um teste de VIH uma semana depois de ter sexo dirá a uma pessoa se ele ou ela têm VIH.

17. Uma rapariga pode ficar grávida na primeira vez que tem relações sexuais.

18. O vírus do HIV pode transmitir-se através do sexo oral desprotegido.

19. A pílula previne contra as infeções sexualmente transmissiveis (IST's).

20. Um adolescente não precisa de autorização dos pais para pedir o preservativo ou a pílula num Centro de Saúde ou noutras consultas de atendimento a jovens.

21. Não há uma idade própria para se iniciar a vida sexual.

22. O sexo é uma forma de prazer.

23. Ter sexo com mais de um parceiro(a) pode aumentar a probabilidade de uma pessoa ser infetada com o $\mathrm{VIH}$.

24. Qualquer aconselhamento na área da sexualidade que aconteça na escola deve ser dado a conhecer aos encarregados de educação.

25. A única forma de evitar a transmissão do VIH numa relação sexual é o uso do preservativo. 